Supplement of Geosci. Model Dev., 11, 4451-4467, 2018

https://doi.org/10.5194/gmd-11-4451-2018-supplement

(C) Author(s) 2018. This work is distributed under

the Creative Commons Attribution 4.0 License.

(c) (1)

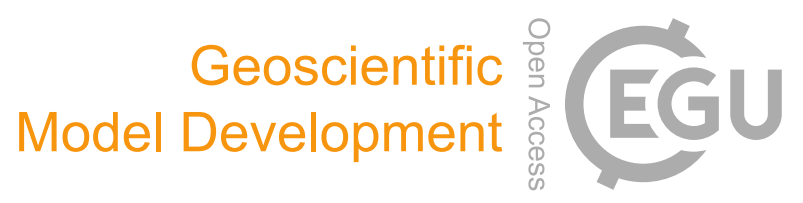

Supplement of

\title{
Implementing spatially explicit wind-driven seed and pollen dispersal in the individual-based larch simulation model: LAVESI-WIND 1.0
}

Stefan Kruse et al.

Correspondence to: Stefan Kruse (stefan.kruse@ @awi.de)

The copyright of individual parts of the supplement might differ from the CC BY 4.0 License. 


\section{Supplement}

\subsection{Verification of wind-dependency}

\subsubsection{Simulation setup}

The simulation experiments were conducted on a 200 x $200 \mathrm{~m}$ plot using the model with the new processes for verification. Populations were initiated on empty areas by randomly distributing a fixed number of seeds during the first 100 years of a 1,000 year long stabilisation period. The simulation model randomly drew weather conditions for each year from the complete available period 1934-2013 during the stabilisation period. In the final 80 simulation years, the actual weather data were used. First, we performed simulation experiments with constant wind conditions to verify the implemented dispersal processes. Wind forcing was from the north or from the south, both with a constant wind speed of $10 \mathrm{~km} \mathrm{~h}^{-1}$. Simulations were repeated 50 times with an input of 250 seeds per year during initialisation.

Second, we evaluated the functionality of the seed dispersal function by forcing the model with wind data from the reanalysis data set ERA-Interim (Balsamo et al., 2015). Simulations were repeated 10 times and population growth initiated by introducing 100 seeds per year during initialisation.

The dispersal distance and direction as well as the release height of every $100^{\text {th }}$ seed dispersal event were recorded during each year of the complete simulation run. Pollination was assessed by recording the distance and direction from the selected pollen sources to the seed positions prior to seed release of all tree individuals present at the final year of the simulation.

\subsubsection{Evaluation of dispersal processes}

The seed dispersal and pollination distances were evaluated by calculating the mean dispersal distances for both processes in bins of $30^{\circ}$ cardinal directions over the complete simulation run for years with available wind data (1979-2012). In addition, we selected two years with contrasting wind patterns, the year 1990 with predominant winds from the east, and 1998 from the west. The results were qualitatively compared to observed values from the literature.

During the simulations, we recorded only one pollination event per tree per year reaching the central area of $20 \mathrm{x} 20 \mathrm{~m}$ of the plot and every $100^{\text {th }}$ seed dispersal event.

\subsubsection{Analyses}

We verified the wind-dependent seed dispersal by comparing the simulation results with the simulated results from the implemented dispersal function and refer to these values as 'expected'. Therefore, we estimated the dispersal distances with the implemented dispersal functions (seeds Eq.4 and pollen Eq.3) under the same conditions as used for driving the model, namely winds from north or south with a constant speed of $10 \mathrm{~km} \mathrm{~h}^{-1}$. The observed mean log-transformed (to achieve normal distribution) dispersal distances were compared to each other with a Welch two-sample t-test. We compared simulated to expected dispersal distances in each wind direction in height classes of trees in $10 \%$ steps of the sorted values, excluding the 
minimum and maximum values where only few observations could be recorded. The simulated data values in the range of $\pm 5 \%$ at the determined height classes were first tested for an interaction between the release height and the dispersal distance with a Spearman's rank-based measure of association (rho) and a t-test. Then we compared the simulated distances for each height class to the expected distances, both log-transformed prior to analysis, with a t-test. Furthermore, we compared the dispersal in both directions to each other using the same procedure.

The simulated pollination distances were compared to estimates from the implemented function under the same conditions, namely the adult tree's position and constant north or south winds with a speed of $10 \mathrm{~km} \mathrm{~h}^{-1}$. The resulting three-dimensional pollination distribution probabilities at simulation year 2011 (first year of fieldwork) were binned to cardinal directions in steps of $30^{\circ}$, starting with north between $345^{\circ}$ and $15^{\circ}$. The simulated and directly estimated log-transformed pollination distances were tested for differences in each individual direction with a Welch two-sample t-test for cases with $>10$ observations. We tested whether the mean of the p-values is significantly greater than 0.01 with a Student t-test.

\subsubsection{Results}

All simulated values were highly correlated to the directly estimated distances ( $p>0.05$, Table S2; Fig. 2). The mean logtransformed simulated distances in a north or south direction were not different when comparing releasing trees of the same height ( $\mathrm{p}>0.05$, Table S3).

The distances, simulated and expected from direct calculation, are more similar for those pollination directions with more samples, and only 4-6\% of tests show significant differences (Table S4). The higher distance values for these directions may be based on the uniform sampling of fathers and the associated smaller sample sizes in these directions. In all comparisons, we find no evidence that the differences are significant (Table S5).

Table S1. Model structures and memory consumption as the estimated mean over a typical dense forest plot of one hectare, with 80 years climate forcing data and an additional 1,000 years initialising phase.

\begin{tabular}{|c|c|c|c|}
\hline Structure & Substructure & $\begin{array}{r}\text { Total for each } \\
\text { element in structure } \\
{[\mathrm{b}]}\end{array}$ & $\begin{array}{r}\text { Total in } \\
\text { each simulation } \\
{[\mathrm{kb}]}\end{array}$ \\
\hline Parameter & - & 642 & 0.64 \\
\hline Weather ${ }^{1}$ & Year(i) & 390 & 31.2 \\
\hline Environment $^{2}$ & Grid(i) & 54 & 2,700 \\
\hline Trees $^{3}$ & Tree(i) & 120 & 3,000 \\
\hline Seeds $^{4}$ & Seed(i) & 98 & 9,016 \\
\hline Evaluation $^{5}$ & Evaluation(i) & 117 & 105.86 \\
\hline TOTAL & & & $14,853.7$ \\
\hline
\end{tabular}

1: with 80 years of weather input data on a monthly basis;

2: 50,000 grid tiles at a resolution of $20 \times 20 \mathrm{~cm}$ tiles on a hectare;

3: 25,000 larch individuals appear per hectare in simulations of a typical dense forest plot;

4: $~ 92,000$ larch seeds are present on a hectare of a typical dense forest plot simulation;

5: the length of the simulation is calculated with 1,000 years stabilization and 80 years simulation phase 
Table S2. Comparison of simulated to directly estimated seed dispersal from the implemented functions of north and south winds at the same observed release heights calculated for each direction separately.

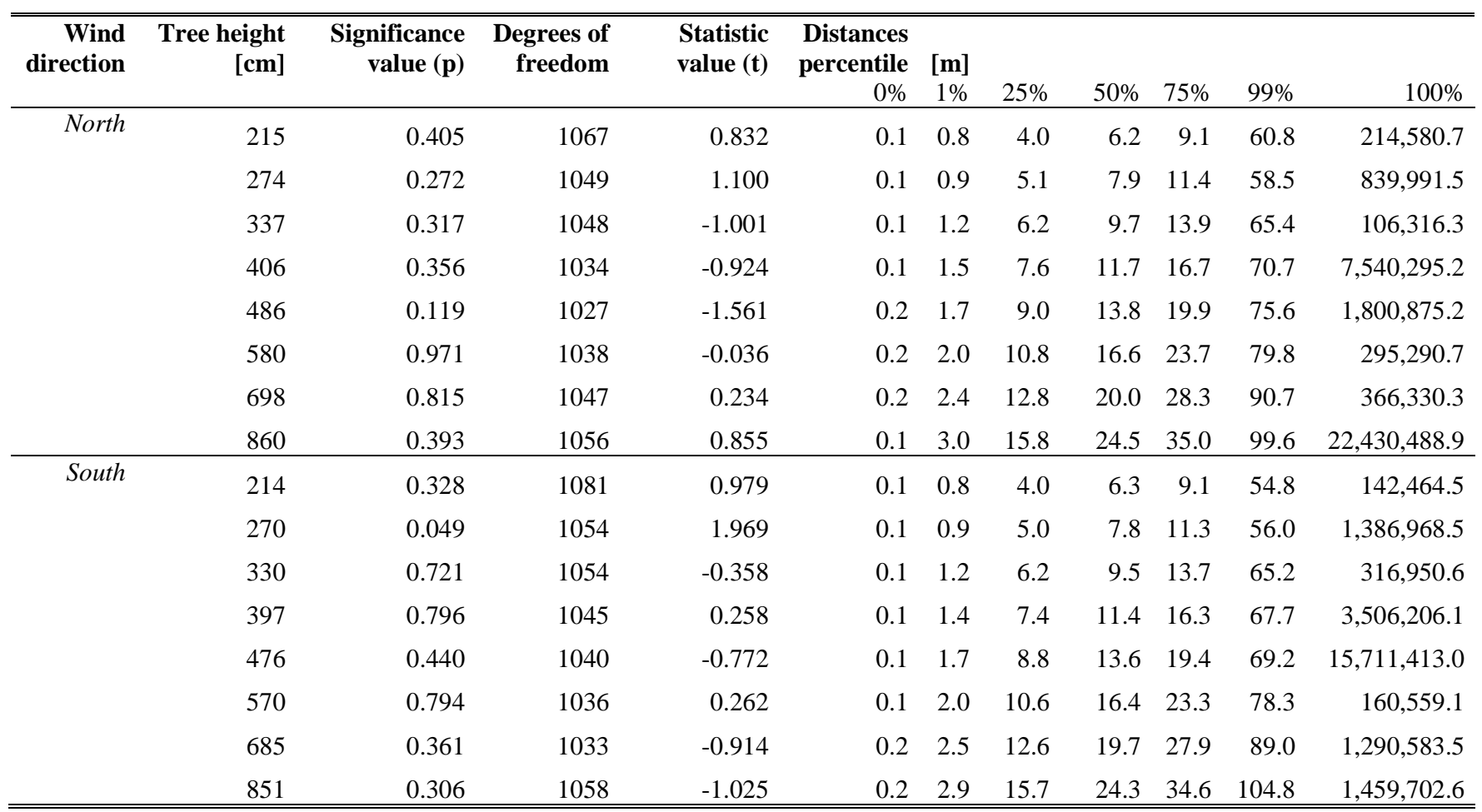


Table S3. Comparison of seed dispersal distances in simulations forced with winds from north and south directions.

\begin{tabular}{|c|c|c|c|c|c|c|c|c|c|c|c|}
\hline $\begin{array}{r}\text { Wind } \\
\text { direction }\end{array}$ & $\begin{array}{r}\text { Tree height } \\
{[\mathrm{cm}]}\end{array}$ & $\begin{array}{r}\text { Distances } \\
\text { percentile } \\
0 \% \\
\end{array}$ & $\begin{array}{r}{[\mathrm{m}]} \\
1 \% \\
\end{array}$ & $25 \%$ & $50 \%$ & $75 \%$ & $99 \%$ & $100 \%$ & $\begin{array}{r}\text { Significance } \\
\text { value }(p)\end{array}$ & $\begin{array}{r}\text { Degrees of } \\
\text { freedom }\end{array}$ & $\begin{array}{l}\text { Statistic } \\
\text { value }(t)\end{array}$ \\
\hline North & 215 & 0.1 & 0.7 & 4.0 & 6.2 & 9.0 & 60.2 & $214,580.7$ & 0.336 & 58,956 & -0.961 \\
\hline South & & 0.1 & 0.8 & 4.0 & 6.3 & 9.1 & 55.9 & $142,464.5$ & & & \\
\hline North & 272 & 0.1 & 0.9 & 5.1 & 7.8 & 11.3 & 58.8 & $94,025.3$ & 0.235 & 88,336 & -1.189 \\
\hline South & & 0.1 & 1.2 & 6.2 & 9.6 & 13.9 & 67.1 & $316,950.6$ & & & \\
\hline North & 402 & 0.1 & 1.4 & 7.5 & 11.6 & 16.5 & 71.2 & $7,540,295.2$ & 0.164 & 100,519 & 1.393 \\
\hline South & & 0.1 & 1.4 & 7.4 & 11.5 & 16.4 & 68.6 & $2,260,443.8$ & & & \\
\hline North & 481 & 0.2 & 1.7 & 8.9 & 13.7 & 19.7 & 73.8 & $1,800,875.2$ & 0.388 & 101,366 & 0.864 \\
\hline North & 692 & 0.2 & 2.4 & 12.7 & 19.8 & 28.1 & 91.8 & $366,330.3$ & 0.230 & 92,599 & -1.201 \\
\hline South & & 0.2 & 2.5 & 12.8 & 19.9 & 28.2 & 89.8 & $1,290,583.5$ & & & \\
\hline North & 856 & 0.1 & 3.0 & 15.7 & 24.4 & 34.8 & 99.3 & $22,430,488.9$ & 0.921 & 74,922 & 0.100 \\
\hline South & & 0.2 & 2.9 & 15.7 & 24.5 & 34.7 & 105.8 & $1,459,702.7$ & & & \\
\hline
\end{tabular}

Table S4. Pollination event comparison between simulated and expected values. The statistics are based on 50 simulation repeats. Significant differences between expected and simulated distances tested with a Welch two-sample t-test.

\begin{tabular}{lllrr}
\hline \hline $\begin{array}{l}\text { Cardinal } \\
\text { direction } \\
\text { of winds }\end{array}$ & $\begin{array}{l}\text { Direction of } \\
\text { pollination }\left[^{\circ}{ }^{\circ}\right.\end{array}$ & $\begin{array}{l}\text { Distance } \\
\text { (simulated/expected) }[\mathbf{m}]\end{array}$ & $\begin{array}{r}\text { Fraction of observations } \\
\text { (simulated/expected) [\%] }\end{array}$ & $\begin{array}{r}\text { Fraction of } \\
\text { significant } \\
\text { differences }\end{array}$ \\
\hline North & $135-165$ & $44.32 / 43.79$ & $9.00 / 8.39$ & $20 \%$ \\
& $165-195$ & $39.03 / 38.90$ & $81.53 / 82.68$ & $4 \%$ \\
& $195-225$ & $40.03 / 40.19$ & $9.47 / 8.93$ & $14 \%$ \\
\hline South & $315-345$ & $40.94 / 40.47$ & $7.55 / 7.92$ & $12 \%$ \\
& $345-15$ & $36.98 / 37.90$ & $82.92 / 82.8$ & $6 \%$ \\
& $15-45$ & $42.76 / 40.84$ & $9.51 / 9.28$ & $22 \%$ \\
& $45-75$ & $53.80 /-$ & $0.01 / 0.00$ & $*$ \\
\hline
\end{tabular}

* only one observation, thus excluded from further analyses. 
Table S5. Comparison of pollination dispersal distances with Student's t-test of the mean p-values at a significance level of 0.01 . Only cases with $>10$ pollination events of the five trees producing most seeds were considered.

\begin{tabular}{llrrrr}
\hline \hline $\begin{array}{l}\text { Cardinal direction } \\
\text { of winds }\end{array}$ & $\begin{array}{l}\text { Wind } \\
\text { direction }\left[^{\circ}\right]\end{array}$ & Mean p-value & $\begin{array}{r}\text { Significance } \\
\text { value (p) }\end{array}$ & $\begin{array}{r}\text { Degrees } \\
\text { of } \\
\text { freedom }\end{array}$ & $\begin{array}{r}\text { Statistic } \\
\text { value (t) }\end{array}$ \\
\hline North & $165-195$ & 0.449 & $<0.001$ & 49 & 10.133 \\
& $135-165$ & 0.277 & $<0.001$ & 38 & 5.629 \\
& $195-225$ & 0.406 & $<0.001$ & 37 & 6.704 \\
\hline South & $345-15$ & 0.415 & $<0.001$ & 49 & 10.171 \\
& $15-45$ & 0.302 & $<0.001$ & 40 & 5.734 \\
& $315-345$ & 0.408 & $<0.001$ & 39 & 8.315 \\
\hline \hline
\end{tabular}


Table S6. Test statistics for generalised nonparametric regression analyses (significance level: $* * * \mathbf{p}<0.001$ ).

\begin{tabular}{|c|c|c|c|c|c|c|c|c|}
\hline \multirow{3}{*}{$\begin{array}{l}\text { Simulation version } \\
\text { +POLLEN_PAR A }\end{array}$} & \multirow{2}{*}{$\begin{array}{l}\text { Model formula } \\
t \sim N t\end{array}$} & \multirow{2}{*}{$\begin{array}{r}\begin{array}{r}\text { Aikaike's } \\
\text { Information } \\
\text { Criterion } \\
\text { (AIC) }\end{array} \\
964\end{array}$} & \multirow{2}{*}{$\begin{array}{r}\begin{array}{r}\text { Dispersion } \\
\text { parameter for } \\
\text { Gaussian family }\end{array} \\
0.17\end{array}$} & \multirow[t]{2}{*}{$\begin{array}{r}\text { Model term } \\
N t\end{array}$} & \multirow{3}{*}{$\begin{array}{r}\begin{array}{r}\text { Degrees } \\
\text { of }\end{array} \\
\text { freedom }\end{array}$} & \multirow{3}{*}{$\begin{array}{r}\begin{array}{r}\text { Sum of } \\
\text { squares }\end{array} \\
10160.4 \\
184.2\end{array}$} & \multicolumn{2}{|c|}{$\begin{array}{l}\text { F-test Significance } \\
\text { statistic } \\
\text { value }\end{array}$} \\
\hline & & & & & & & 59340.3 & $* * *$ \\
\hline & & & & Residuals & & & & \\
\hline & $t \sim N s$ & 1042 & 0.29 & $N s$ & 1 & 9093.8 & 31780.8 & $* * *$ \\
\hline & & & & Residuals & 1076 & 307.9 & & \\
\hline & $t \sim N t+N s$ & 1167 & 0.15 & $N t$ & 1 & 9879.2 & 65019.1 & $* * *$ \\
\hline & & & & $N s$ & 1 & 7.3 & 48.2 & $* * *$ \\
\hline & & & & Residuals & 1072 & 162.9 & & \\
\hline & $t \sim N t+N s+N t: N s$ & 1722 & 0.14 & $N t$ & 1 & 10688.2 & 75655.2 & $* * *$ \\
\hline & & & & $N s$ & 1 & 705.7 & 4995.0 & $* * *$ \\
\hline & & & & $N t: N s$ & 1 & 1843.4 & 13048.4 & $* * *$ \\
\hline & & & & Residuals & 1071 & 151.3 & & \\
\hline \multirow[t]{11}{*}{ +POLLEN } & $t \sim N t$ & 884 & 0.17 & $N t$ & 1 & 9730.8 & 56047.3 & $* * *$ \\
\hline & & & & Residuals & 1077 & 187.0 & & \\
\hline & $t \sim N s$ & 975 & 0.29 & $N s$ & 1 & 8857.5 & 30113.9 & $* * *$ \\
\hline & & & & Residuals & 1077 & 316.8 & & \\
\hline & $t \sim N t+N s$ & 1183 & 0.14 & $N t$ & 1 & 9472.3 & 66387.8 & $* * *$ \\
\hline & & & & $N s$ & 1 & 9.0 & 63.3 & $* * *$ \\
\hline & & & & Residuals & 1073 & 153.1 & & \\
\hline & $t \sim N t+N s+N t: N s$ & 1754 & 0.13 & $N t$ & 1 & 10215.8 & 77934.1 & $* * *$ \\
\hline & & & & $N s$ & 1 & 582.7 & 4445.4 & $* * *$ \\
\hline & & & & $N t: N s$ & 1 & 1647.4 & 12567.5 & $* * *$ \\
\hline & & & & Residuals & 1072 & 140.5 & & \\
\hline \multirow[t]{11}{*}{ +POLLEN_PAR B } & $t \sim N t$ & 734 & 0.15 & $N t$ & 1 & 9923.8 & 64828.7 & $* * *$ \\
\hline & & & & Residuals & 1075 & 164.6 & & \\
\hline & $t \sim N s$ & 833 & 0.25 & $N s$ & 1 & 8939.3 & 35072.6 & $* * *$ \\
\hline & & & & Residuals & 1075 & 274.0 & & \\
\hline & $t \sim N t+N s$ & 1045 & 0.13 & $N t$ & 1 & 9583.7 & 76495.4 & $* * *$ \\
\hline & & & & $N s$ & 1 & 20.8 & 166.2 & $* * *$ \\
\hline & & & & Residuals & 1071 & 134.2 & & \\
\hline & $t \sim N t+N s+N t: N s$ & 1596 & 0.11 & $N t$ & 1 & 10405.4 & 91086.0 & $* * *$ \\
\hline & & & & $N s$ & 1 & 681.4 & 5964.8 & $* * *$ \\
\hline & & & & $N t: N s$ & 1 & 1705.2 & 14927.1 & $* * *$ \\
\hline & & & & Residuals & 1070 & 122.2 & & \\
\hline \multirow[t]{11}{*}{ SEED } & $t \sim N t$ & -403 & 0.05 & $N t$ & 1 & 1440.4 & 30730.1 & $* * *$ \\
\hline & & & & Residuals & 1075 & 50.4 & & \\
\hline & $t \sim N s$ & -375 & 0.06 & $N s$ & 1 & 1317.6 & 21817.7 & $* * *$ \\
\hline & & & & Residuals & 1075 & 64.9 & & \\
\hline & $t \sim N t+N s$ & -233 & 0.04 & $N t$ & 1 & 1396.3 & 34099.6 & $* * *$ \\
\hline & & & & $N s$ & 1 & 9.4 & 229.9 & $* * *$ \\
\hline & & & & Residuals & 1071 & 43.9 & & \\
\hline & $t \sim N t+N s+N t: N s$ & 40 & 0.04 & $N t$ & 1 & 1512.3 & 37915.5 & $* * *$ \\
\hline & & & & Ns & 1 & 37.9 & 950.9 & $* * *$ \\
\hline & & & & $N t: N s$ & 1 & 288.9 & 7243.1 & $* * *$ \\
\hline & & & & Residuals & 1070 & 42.7 & & \\
\hline
\end{tabular}

